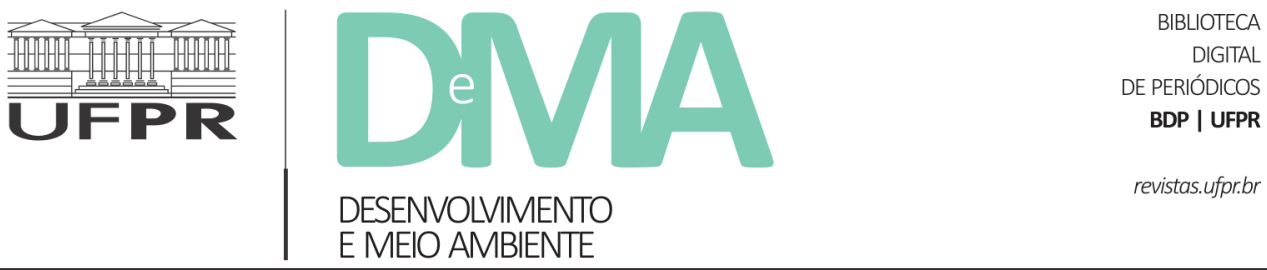

\title{
Patagonia Chilena: conflictos territoriales y procesos de conservación ambiental en el siglo XXI
}

\section{The Chilean Patagonia: territorial conflicts and environmental conservation in the $21^{\text {ST }}$ century}

\section{A Patagonia Chilena: conflitos territoriais e os processos de conservação ambiental no século XXI}

\author{
Juan Carlos Rodríguez TORRENT ${ }^{1}$, Janett Vallejo ROMAN²*, Nicolas Gissi BARBIERI ${ }^{3}$ \\ ${ }^{1}$ Escuela de Diseño, Universidad de Valparaíso, Valparaíso, Chile. \\ ${ }^{2}$ Centro de Investigaciones y Estudios Superiores en Antropología Social, Unidad Regional Golfo (Ciesas-Golfo), Xalapa, Veracruz, México. \\ ${ }^{3}$ Universidad de Chile, Santiago, Chile. \\ *E-mail de contacto: janettvallejo@gmail.com
}

Artículo recibido el 2 de enero, 2020, versión final aceptada el 24 de abril, 2021, publicado el 22 de septiembre de 2021.

RESUMEN: Se presenta un análisis de carácter antropológico sobre procesos de reescritura territorial en la Patagonia chilena, específicamente en la región de Aysén. La tesis central sostiene que la presencia de nuevos actores no estatales, con propiedad de la tierra y de ecosistemas, y con orientaciones filosóficas de la ecología profunda, genera nuevas condiciones para la conservación ambiental y redefine la posición de los habitantes tradicionales, productores ligados a la ganadería familiar. Se plantea, críticamente, que los nuevos lenguajes de valoración operan como una expresión política de poder y autoridad, que impacta severamente en la posibilidad de reproducción de las unidades domésticas, las que necesitan de amplios espacios alimenticios para su ganado y del bosque nativo para protección. De este modo, el espacio tradicional de la vida cotidiana pierde regularidad por la creación de parques privados, ya que tienen fines no productivos e incorporan valores post-humanistas. Asimismo, a través de registros etnográficos, se constata un proceso gradual de desnacionalización territorial, de ausencia de soberanía local y dificultades para la reproducción de la cultura rural de la Patagonia, que complejizan imaginar el futuro por parte de las unidades productivas.

Palabras clave: ruralidad; naturaleza; ecología profunda; conservación ambiental; Patagonia. 
ABSTRACT: An anthropological analysis of territorial rewriting processes in Chilean Patagonia, specifically in the Aysén region, is presented. The central thesis maintains that the presence of new non-state actors, with ownership of the land and ecosystems, and with philosophical orientations of deep ecology, generates new conditions for environmental conservation and redefines the position of traditional inhabitants, producers linked to family farming. It is critically posited that the new valuation languages operate as a political expression of power and authority, which severely impacts the possibility of reproduction of domestic units, which need ample feeding spaces for their livestock and the native forest for protection. In this way, the traditional space of daily life loses regularity due to the creation of private parks, since they have non-productive purposes and incorporate post-humanist values. Likewise, through ethnographic records, a gradual process of territorial denationalization, absence of local sovereignty and difficulties for the reproduction of the rural culture of Patagonia is verified, which make it difficult to imagine the future by the productive units.

Keywords: rurality; nature; deep ecology; environmental conservation; Patagonia.

RESUMO: Apresenta-se uma análise de cunho antropológico sobre processos de um reescrever territorial na Patagônia chilena, especificamente na região de Aysén. A tese central sustém que a presença de novos atores não estatais com posse da terra e dos ecossistemas, e com orientações filosóficas de profunda ecologia, geram novas condições de conservação ambiental e redefine a posição dos habitantes tradicionais, produtores vinculados à pecuária familiar. Enfoca-se criticamente em novas linguagens de valoração que operam como expressão política de poder e autoridade, e que impacta severamente na possibilidade de reprodução das unidades domésticas que precisam de amplos espaços de alimentação para seus rebanhos, assim como da mata nativa para sua proteção. Sendo assim, o espaço tradicional da vida cotidiana perde força com a criação de parques privados, uma vez que estes possuem finalidades improdutivas e incorporam valores pós-humanistas. Da mesma forma, por meio de registros etnográficos, verifica-se um processo gradativo de desnacionalização territorial, de ausência de soberania local e dificuldades para a reprodução da cultura rural patagônica, sendo complexo imaginar o futuro por parte das unidades produtivas.

Palavras-chave: ruralidade; natureza; ecologia profunda; conservação ambiental; Patagônia.

\section{Introducción}

¿Cuál es la relación entre un taller artesanal de los años 60's, ubicado en un garaje del condado de Ventura en California, Estados Unidos, en que se confeccionaban artículos para la práctica deportiva de montaña, tablas de surf y una furgoneta equipada para viajar, y lo que acontece territorialmente cinco décadas más tarde, en la Patagonia chilena (y argentina)? La pregunta tiene un origen lejano, pero en la actualidad la respuesta nos ayuda a pensar la gobernanza actual de la Patagonia, en relación a las tensiones entre actores productivos y no producti- vos, o profesantes de una filosofía verde y la vida cotidiana de poblaciones de tradición ganadera.

Cuatro jóvenes, en 1968, alejándose de la experiencia traumática de su generación con la intervención norteamericana en Vietnam, emprendieron un viaje de 16.000 kilómetros hacia "el sur", al monte Fitz Roy; hacia los paisajes humanos del subdesarrollo y el espacio abierto de la naturaleza del fin del mundo, lo que -posteriormente- permitió la transformación de toda su experiencia de vida, ya que el propósito del viaje no estuvo en encontrar nuevos caminos, sino en introducir nuevas miradas. En este viaje, iban Roy Gear, fundador de la 
compañía textil Patagonia; y, Douglas Tompkins, fundador de The North Face y Espirit (ver Mountain of storns y $180^{\circ}$ south). El más reconocido en Chile ha sido Tompkins, quien ya había estado en 1961 en el sur de Chile y Argentina. Tuvo una vida sin sobresaltos, sus padres fueron coleccionistas de arte y urbanistas, de niño y adolescente fue escalador y conoció las tablas de esquí; mochilero y aventurero en el valle de Yosemite, con juvenil adhesión a grupos pacifistas. Tempranamente millonario, y "aburrido de ganar dinero", buscó otros incentivos para su vida, saliendo de la vida pública y del "mundo civilizado", para concentrarse en la reflexión y la conservación de tierras (Sánchez, 2006, p. 69-112).

Desde ahí cuestionó el arquetipo de las razones para vivir de occidente: la confianza ilimitada en las capacidades humanas y su expansión a todos los confines de la naturaleza a través de la idea de desarrollo.

Su presencia marca la entrada de la Patagonia al siglo XXI. Filosóficamente disputa la producción de sentido sobre la naturaleza y la vida social, al descubrir que la geografía agreste del sur austral generó un espacio subnacional, que no tuvo gran contacto con el resto de Chile, donde existen ecosistemas impolutos, y otros que aún puede recuperarse, salvarse y restituirse. Que aún existe flora y fauna endémica que en algunas zonas pueden vivir con su propio ritmo; y, en lo íntimo, que él puede ayudar a profundizar esta condición a través de la compra de tierras, gestionando, a través de distintas fundaciones, la figura de parques y reservas privadas para su protección y recuperación.
Elabora un concepto básico en términos territoriales, con uso de energías locales, desterrando todo aquello que atente contra la biodiversidad y que altere el clima. Desarrolla la belleza como factor de equilibrio de una vida integrada, a través del respeto hacia todos los seres y sin jerarquías, dentro de una concepción filosófica de la ecología profunda. Ve, en este paraje que cobija, un refugio, un mundo de posibilidades para dotar de sentido su propia existencia y generar una propuesta y modelo de relaciones diversas entre humanos y no humanos ${ }^{1}$.

En lo que sigue, a partir de registros de campo e información secundaria, planteamos como principal reflexión: que Douglas Tompkins se convierte en un incumbente en el destino de la Patagonia y en la construcción de la imagen de hipervaloración que para los chilenos y el mundo significa. Sin embargo, su razón de ser y ímpetu filosófico, que implica control de grandes porciones de territorio, afecta de manera radical la geografía de la vida cotidiana, del trabajo y los ciclos reproductivos de los lugareños, que durante generaciones ha dado forma al territorio austral y a las prácticas de vida locales a través de la ganadería extensiva, limitando su propia reproducción social y biológica. Esta presencia define una relación litigante con la población local, porque su discurso ecológico y político permite posicionar un imaginario extralocal sobre nuevos valores de uso sobre estas tierras, y sobre su flora y fauna endémicas, generando una adhesión favorable y anti extractivista en la opinión pública nacional e internacional.

\footnotetext{
${ }^{1}$ Ver: https://albertofraile.net/entrevista-douglas-tompkins/; https://www.revistaenfoque.cl/douglas-tompkins-el-capitalismo-es-una-amenaza-real-para-el-planeta
} 


\section{Antecedentes: Douglas Tompkins, el filántropo y ambientalista}

Como filántropo, ambientalista y seguidor de la ecología profunda, su pensamiento se inscribe en una controversia permanente: cómo la naturaleza es construida, y cómo puede ser representada, reclamada e imaginada, ya que es siempre un recurso retórico y simbólico usado por las instituciones financieras (especulación), las empresas privadas (costos y beneficios), las instituciones del Estado (normas, reglamentos, acuerdos nacionales e internacionales), las comunidades (modos tradicionales de actuación) y las familiares (apreciaciones objetivas y subjetivas), que representan una diversidad de participantes, posiciones, acciones, información, control, resultados potenciales, costos y beneficios de distinto orden (Ostrom \& Walker, 2003; Ostrom, 2013). Introduce en la Patagonia un punto de vista crítico sobre la presión hacia los grandes bosques, la desforestación, el cambio climático y lo que significa la masiva extinción de especies, como productos de la revolución industrial y el sobredesarrollo, que nos llevan al abismo de la historia, en la medida que sin biodiversidad todo es irrelevante (ver: https:// www.youtube.com/watch?v=In2bdaN3QdI).

Aunque son pocos los escritos y escasas las entrevistas concedidas en las que socializa su pensamiento, comunicando más sus posiciones a través de las publicaciones de sus fundaciones, su propuesta está definida dentro de los marcos de la "ecología profunda". Siguiendo los planteamientos de Arne Naess (1995) y George Sessions (1987), por una parte, destaca el descentramiento del antropocentrismo, y la superación del binarismo cultura-naturaleza, para reconocer la multiplicidad de las existencias; y, por otra, perfila una crítica hacia la figura del progreso y al modelo civilizatorio occidental dominante, postulando a un imaginario de encadenamiento de la vida en común, dentro de una perspectiva holística o ecosófica del derecho a la existencia.

El "vive y deja vivir" configura un ecocentrismo, que implica coexistir y cooperar en interrelaciones complejas a nivel de individuos, especies, poblaciones, hábitats, así como culturas humanas y no-humanas. Un ser, cualquiera sea, lo es siempre en relación con los demás y con lo demás. De ahí que, la idea central de biofilia ayuda a dar forma a la existencia colectiva; imponiéndose esta concepción a las áreas bajo control de sus fundaciones, afianzando el idéntico derecho que tiene cada ser a existir y prosperar, y el reconocimiento del valor de todos y cada uno de los vivientes. Por tanto, si no existe un utilitarismo en favor de lo humano, la forma cómo pensamos incide en la forma cómo nos relacionamos con la naturaleza, tal como está presente en el primer postulado de la ecología profunda: El florecimiento y el bienestar de la vida humana y no-humana en la Tierra poseen un valor por sí mismos. Estos valores no dependen de la utilidad del mundo no-humano para los objetivos humanos ${ }^{2}$.

\footnotetext{
${ }^{2}$ Ver: https://www.endemico.org/principios-de-la-ecologia-profunda/\#: :text=Los $\% 20$ principios $\% 20 \mathrm{de} \% 201 \mathrm{la} \% 20 \mathrm{ecolog} \% \mathrm{C} 3 \% \mathrm{ADa}$,un $\% 20$ llamamiento $\% 20$ al $\% 20$ activismo $\% 20$ ecologista.\&text=En $\% 20$ s $\%$ C3\%ADntesis $\% 2$ C $\% 20$ se $\% 20$ propone $\% 20$ que,ser $\% 20 \mathrm{~m} \% \mathrm{C} 3 \% \mathrm{~A} 1 \mathrm{~s} \% 20$ ecos $\%$ C3\%B3fico $\% 20$ que $\% 20$ ecol $\%$ C3\%B3gico
} 
La idea fuerza de un campo total o relacional, de interdependencia e interconexión entre todo lo real, esclarece cómo consideramos a la naturaleza; si solo como un simple contenedor de riqueza, o un ámbito misterioso, lo que desafía la recurrencia de los modos tradicionales de relación con ella. Así, el compromiso social, ambiental y político dentro del amplio espectro de lo que se denomina "verde", es de una economía moral, y toma distancia del desarrollismo en su doble connotación: a) la economía moderna e industrial, y b) la reducción de la pobreza y la mejora de los índices de bienestar a través de ésta. También de cuestiones variadas como el "eco-socialismo", el "desarrollo endógeno", la "modernidad reflexiva" e incluso la "sustentabilidad", ya que una entrada en la WEB ofrece un número inabordable de lecturas y posibilidades de entendimiento sobre "lo sostenible", persistiendo las dificultades sobre su significado (metas) y las condiciones necesarias para que se realice (principios).

La experiencia personal de la naturaleza telúrica de los bosques milenarios, los cursos de agua libre, la flora y fauna endémica, sumado a su desencanto por las prácticas de la modernidad, se imponen como lectura para transformar las coordenadas geográficas de una bio-región, en apariencia casi despoblada y entregada tempranamente por el Estado a la crianza de ovejas y vacunos a empresarios particulares (1903-1905) ${ }^{3}$. Entiende que, toda la población que configuró la sociedad patagónica moderna del siglo XX, estuvo conformada por individuos o grupos inscritos en la cadena de producción ovina extensiva, lo que significó la degradación de suelos: mientras más ovejas, más bosque había que talar. Y, que además, se trataba de un proceso económico y de poblamiento especialmente vulnerable a la depresión del comercio internacional de la lana, haciendo insostenible la mantención de un masa ganadera que en algunas estancias superaba las 500.000 cabezas.

La ocupación de la Patagonia "avanzó al paso de los rebaños"; "las ovejas fueron responsables del primer ciclo de colonización"; y, "pusieron a la Patagonia en el mapa" (Coronato, 2010, p. 3-5). Hubo una forma de ocupación con minifundios marginales y no de fundación de asentamientos (op. cit.), por lo que la configuración geográfica actual es resultado del proceso de ganadería ovina extensiva (Vázquez, 2017) y de disputa sobre tierras excedentarias. El proceso de desarrollo de la ganadería se convirtió en la forma más económica y rápida de poner esas tierras en producción, ya que otras formas de incursión territorial hubiesen requerido de préstamos que los gobiernos no estaban en condiciones de otorgar o políticas territoriales difíciles de cumplir.

De este proceso chileno-argentino de producción de ganado, respectivamente con montañas boscosas y mesetas estépicas, surge un apego a la tierra y a los animales, la necesidad de comercializar en el mercado local, de vender a compradores ocasionales y ambulantes, a un productor mayor o un comercializador externo. Se trató de un mundo con poco o nulo acceso al crédito, un bajo poder de negociación de precios, e ingresos irregulares provenientes de estas transacciones y actividades varias, como la comercialización de leña.

\footnotetext{
${ }^{3}$ Se trata de entregas de tierras a las sociedades Industrial de Aysén, Estancia Cisnes y Sociedad Valle Chacabuco, las que fueron favorecidas por el Presidente Germán Riesco (1901-1906). Una situación que fue coincidente con el gravamen impuesto a la carne importada desde Argentina, que favoreció el encarecimiento de la misma, y que en 1905 dio paso a la llamada "huelga de la carne".
} 
Desde fines del siglo pasado, la impronta de Tompkins como vecino que hace territorio y piensa territorio, se hace cargo de este "fracaso" y "mal desarrollo", en cuanto significaba un proceso extractivista de exportación de suelo, sin que mejoraran las condiciones de vida de la población. En una primera lectura se expresa en la franca oposición al desarrollo capitalista de tipo ganadero y cualquier otra posibilidad de corte extractivista ${ }^{4}$; y en una segunda, frente a prácticas regulares de la población rural local como sobrepastoreo y uso de recursos del bosque, con restricciones en tierras que por su abundancia estaban bajo su uso y sobre formas arraigadas en el proceso mismo de colonización.

La cría de ovejas destruyó suelos para que la carne y lanas producidas se disfrutaran en otras regiones. Frente a ello, dos cuestiones están en la base de lectura territorial de Tompkins: 1) varias décadas de sobrepastoreo y desforestación para ampliar cobertura de praderas, donde la capacidad de carga ecosistémica es excedida; y, 2) la sobrestimación inicial de la propia capacidad de carga, adecuada naturalmente a tropillas de camélidos de 30 a 50 ejemplares.

Tompkins apuesta por un tipo de escalas y prácticas diferentes, lo que da forma a una polémica consciencia no vista en la opinión pública nacional, en el Estado y en la élite económica. Adquiere grandes extensiones de tierras para restablecer ecosiste- mas a través de biocorredores, expulsando la fauna introducida (vacunos, caballo, ovejas); perfilándose, además, como el empresario que "viene de vuelta", el descreído de recetas ligadas al desarrollo, ya que conoció la economía, el éxito en el comercio y en los buenos negocios por dentro. Se plantea recuperar y restaurar localmente lo destruido por un régimen de ganadería extensiva. Moviliza un pensamiento que desea frenar el avance del extractivismo, estructurando una noción abstracta del territorio bajo el estatuto de una convicción de sustentabilidad fuerte y radical. Sus posiciones principistas superan todo mito del desarrollo, especialmente al mito del "desarrollo sustentable", por lo que la ciencia posible es la de la sustentabilidad sin jerarquías o al servicio de ésta. Observa con desdén la instrumentalización sistemática de la ciencia al servicio de la dominación humana sobre las condiciones naturales que implica su obrar.

De lo anterior se derivan cuatro ejes programáticos: 1) la necesidad de constituir parques privados de protección a la biodiversidad ${ }^{5}$; 2) la restauración de ecosistemas y reforestación, con la expulsión de fauna exógena con el fin ideal de llegar a una fase anterior de sucesión ecológica; 3) la propuesta de una nueva agricultura en equilibrio con el mundo silvestre; y, 4) el desarrollo de un activismo ambiental.

\footnotetext{
${ }^{4}$ Adhiere explícitamente al movimiento "Patagonia sin represas", el que tiene entre sus finalidades más importantes una oposición a los mega-proyectos hidroeléctricos, así como generar opiniones y argumentos responsables sobre el valor de los ecosistemas patagónicos (http://www. patagoniasinrepresas.cl/final/contenido.php?seccion=nuestracausa).

${ }^{5}$ Aunque se trata de un control sin alambradas, visualiza -como Garret Hardin (1968)- que la estrategia individual de acceso a los recursos, necesariamente ha llevado a la sobre explotación de los recursos compartidos. Ya que la libertad, de alguna manera arruina a todos.
} 


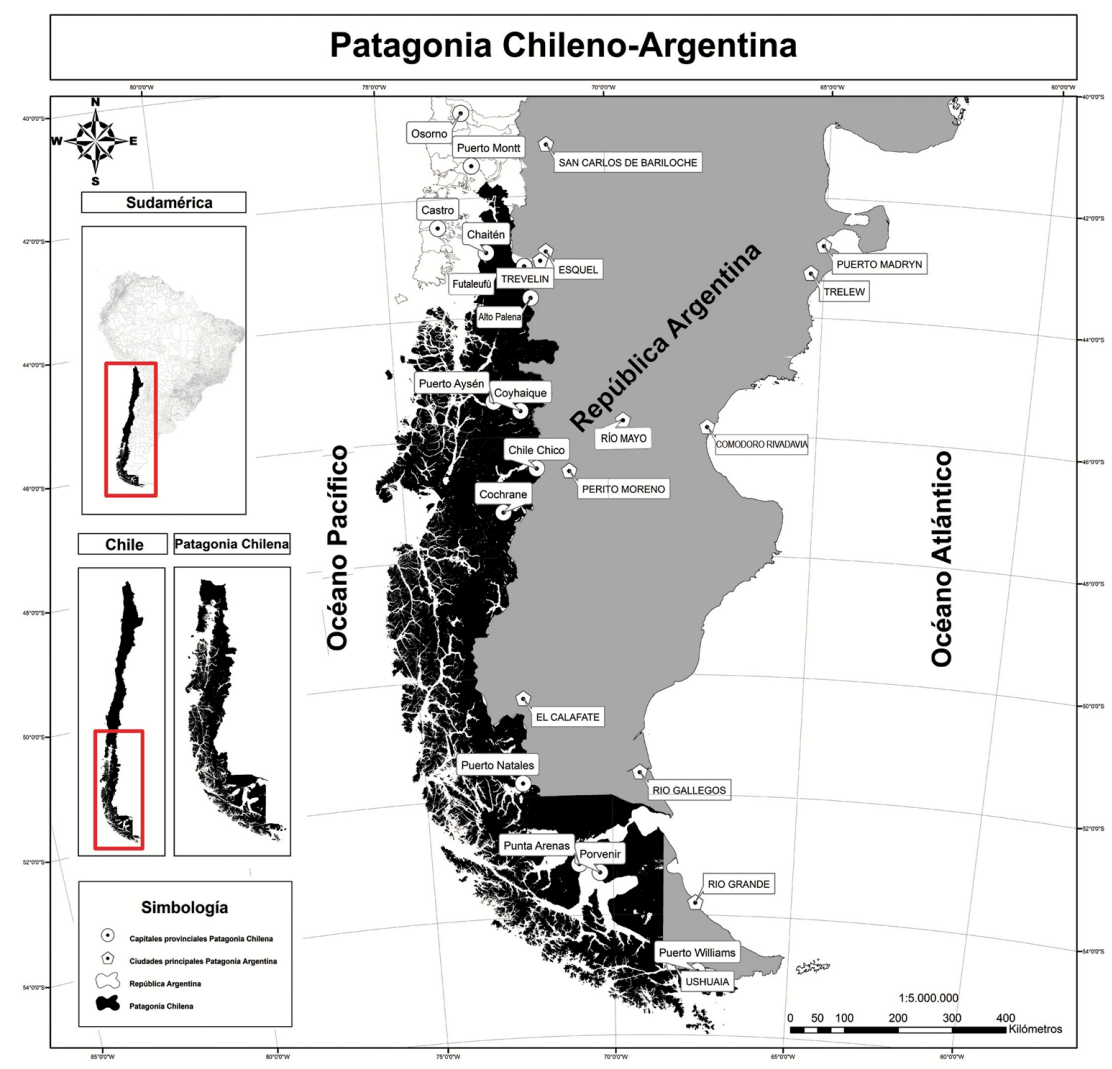

FIGURA 1 - Patagonia chilena y argentina.

FUENTE: Elaborado por los autores conforme a cartografía del Instituto Geográfico Militar.

\section{Aspectos metodológicos}

La metodología utilizada estuvo orientada por la perspectiva cualitativa (Valles, 1999), con énfasis etnográfico y teorización con enfoque antropológico (Grimson et al., 2011). El trabajo de campo se realizó entre los años 2012 y 2018, en el sur de la Región de los Lagos y la región de Aysén, consistiendo en registros etnográficos a partir de un conjunto de observaciones y entrevistas semi-estructuradas en diferentes poblados que no superan los 2.000 habitantes (Futaleufú, Alto Palena, Mañihuales, Puyuhuapi, Cerro Castillo, Chaitén, La Junta, Puerto Cisnes, Cochrane, Villa O’Higgins y Puerto Ibáñez), realizadas a lugareños, administradores de predios y funcionarios públicos. Se incluyó a ganaderos, madereros, pescadores, emprendedores turísticos, transportistas y pequeños comerciantes, considerando el territorio como variable dependiente. El $\mathrm{N}$ de las entrevistas alcanza a 40 . El objetivo de las entrevistas en profundidad y observaciones fue comprender las tensiones entre la ruralidad ganadera 
y maderera y la nueva ruralidad, a partir de diversos avecindamientos (i.e. por amenidad), visualizando el carácter multifuncional que adquiere el territorio a partir de la presencia de nuevos actores y fuerzas nacionales y globales contradictorias de corte proteccionista y extractivista, que reconfiguran las posibilidades locales de reproducción (Ver Núñez et al., 2019; Rodríguez et al., 2018 ). El esquema, asociado también a información secundaria generada en bibliotecas y bases de datos públicos, permitió una triangulación sustentada en un modelo etnográfico multi-localizado, ya que muchas de las explicaciones de lo que está aconteciendo a nivel local se encuentran más allá de los puntos donde se realizaron los registros

\section{Contexto histórico-espacial: ecología profunda y conservación}

La filosofía del equilibrio y la armonía ecológica introduce un nuevo paradigma, donde la naturaleza no sería un fin en sí mismo para los humanos, dejando de ser una entidad concebida como inerte dentro de un régimen capitalista. Entonces, se impone como fundamento en sus territorios privados de casi $900.000 \mathrm{~km}^{2}$ en Chile (Parque Pumalín, Estancia Chacabuco, Parque Yendegalia), y que son parte de 11.5 millones de áreas protegidas, sumado a sus proyectos en Argentina (gran Parque Iberá), introduciendo una nueva coordenada opuesta a las del dualismo cartesiano y diferente a la del ambientalismo más reactivo, que lucha por no degradar y no contaminar.

En lo substantivo, D. Tompkins como vecino incumbente, logra integrar perspectivas posthumanas, es decir, introducir nuevos criterios que implican la deconstrucción normativa de cómo ha sido concebido el ser humano, ya que desestabiliza una concepción que lo identifica como medida de todas las cosas, que lo reconoce en la idea del poder ilimitado y de la inmortalidad (Hayles, 1999; Braidotti, 2015), afincando una materialidad en diálogo que co-fabrica nuevos significados para el territorio. También, posthumano, en la medida que introduce una nueva perspectiva ontológica y umbral civilizatorio, al promover una base alternativa para cuestiones éticas y políticas, y que permiten incorporar otras leyes para que pueda multiplicarse la vida. De modo que, se afianzan localmente nuevas formas de vincularse con los ecosistemas, al abrirse a un nuevo concepto de identidad relacional, que terminan por afectar las posibilidades de reproducción de la agricultura familiar campesina.

\subsection{Las áreas protegidas}

Su fundamentación política y ambiental es prescindente de otras nociones sobre la naturaleza (Descola \& Gísli, 2001; Demerit, 2002) y la existencia (i.e. de la población local), ubicándose en una condición más radical respecto de las áreas protegidas pensadas en términos de ordenamiento, racionalidad y eficiencia del uso de los recursos naturales, la que ha sido la forma clásica de operar e institucionalizar el concepto de conservación (Guha \& Gadgil, 1993; Ferrero, 2018; Agrawal \& Redford, 2009). En lo fundamental, sabemos que las áreas protegidas o de conservación tienen una larga trayectoria en Europa, donde tuvo su origen, siendo descritas como:

"un sistema de medidas sociales, socioeconómicas y técnico-productivas realizadas dentro de los límites 
de un Estado o a escala internacional [principalmente] dirigidas a la utilización racional de los recursos naturales. [para] conservar los recursos naturales, no significa dejar de utilizarlos, sino ordenar su utilización juiciosa, de modo que no se agoten y que se aprovechen de manera perfectamente racional y eficiente" (Del Risco, 1982, p. 169).

En la historia europea, los primeros esfuerzos por conservar tenían un objetivo más político-económico que ambiental, más social, o incluso moral. Bajo una lógica económica, el uso racional y eficiente de la madera, implicaba la generación de planes y proyectos, restricciones y leyes punitivas para quienes hicieran un "uso" diferente al establecido por ciertas élites que determinaron dicha normatividad. Que, como indica Leticia Durand, en el Wilderness Act, de 1964, se les define como "un área donde la tierra y sus comunidades vivientes no son tocadas por el hombre, donde el hombre en sí mismo es un visitante que no permanece" (2017, p. 26), avalando la no intervención antrópica, lo prístino, salvaje e intocable. Lo que se convirtió en el imaginario de la conservación, dando origen a los primeros parques nacionales en Estados Unidos de América $^{6}$ (Santamarina, 2009, p. 308).

En este concepto no es menor, ya que las tierras declaradas parques nacionales, y que pasarían a "resguardo" gubernamental, eran tierras habitadas. En el caso de Yellowstone, por tribus Crow y Shosone, quienes "abandonaron voluntariamente" la región o fueron expulsados por el ejército (Kuppe, 1999, p. 95-96), se introduce un principio de exclusión para construir su carácter. $\mathrm{La}$ justificación de la expulsión se fundamenta en que las extensiones territoriales con alto valor natural debían mantenerse libres de prácticas antrópicas. El estado primigenio o "natural" del espacio era el único medio de protección, por lo que la relación ser humano-Áreas Bajo Protección Ambiental, sólo podía ser a través de la contemplación. Este modelo de conservación que predominó a nivel mundial, excluyó a las poblaciones originarias de sus propias prácticas de conservación y ontologías, diferenciando las ontologías "relacionales" frente a las "secularizadas" o "dualistas", propias del cartesianismo, el capitalismo y el neoliberalismo.

Inicialmente los orígenes de los parques nacionales poseen una connotación usurpadora, pragmática y colonialista (Brockington \& Igoe, 2006). La expulsión y los decretos de expropiación fueron elementos comunes para hacer valer lo que podría ser el "derecho ambiental". De manera que las áreas naturales protegidas se constituyeron como una forma particular de presencia del Estado, definiendo, delimitando y visualizando estos territorios (Ferrero et al., 2013, p. 85).

Este tipo de políticas recibieron fuertes críticas durante el siglo XX, y en las últimas décadas del siglo se planteó de forma sistemática la incorporación de otras variables más allá de las político-económicas y estético-naturales. Por ejemplo, Rivera \& Vallejos-Romero (2015, p. 16), citando a varios autores y diversas perspectivas de análisis, señalan que los principales factores asociados al surgimiento de áreas de conservación provienen de: 1) los recursos disponibles en los distintos países, por lo que la mayor propensión a su existencia se daría en aquellos de mayores recursos y que tienen una población más

${ }^{6}$ El Parque Nacional Yosemite, en 1864 (declarado solo hasta 1890), y el Yellowstone, en 1872. 
educada y sensible hacia valores posmaterialistas; 2) los valores inferiores de las tierras que permiten la adquisición de las mismas; 3) la existencia de condiciones políticas, ya que la discusión en torno a estas zonas compromete políticas públicas, actores políticos y decisiones gubernamentales; 4) que los países que con democracias más sólidas exhiben mejores indicadores de protección; y, 5) la presencia de nuevos actores políticos internacionales, como son las "organizaciones no gubernamentales ambientalistas de influencia global", tales como Conservation International, The Nature Conservancy (TNC), World Wildlife Fund (WWF), Worldwide Fund for Nature, World Resources Institute, tienen también propuestas y voz.

\subsection{Compra de tierras, reterritorialización y conflictos locales}

Douglas Tompkins, tildado por parte de los ganaderos como "el gringo que se viene a quedar con nuestras tierras y aguas", comienza con la adquisición de tierras a propietarios ausentes, en 1991, con la compra del fundo Riñihué (17.000 hectáreas). Más tarde, prosigue al sur de la región de Los Lagos, con lo que constituirían los Parques Pumalín (270.000 hectáreas), Yendegaia (38.750 hectáreas), y la Estancia Chacabuco (81.000 hectáreas), tratando de unir grandes extensiones privadas y las del Estado para configurar un gran parque de la Patagonia $^{7}$ (Figura 2). Posteriormente, ya fallecido (2015), a través de sus fundaciones se convertirá en el principal donante de tierras al Estado de Chile, las que llegarán a unos 4.000.000 millones de hectáreas.

Con las adquisiciones de ecosistemas y paisajes, y la redefinición de su valor patrimonial, así como la adhesión de la ciudadanía nacional e internacional, se produce una completa resimbolización del territorio patagónico en clave de "ecología profunda", que establece marcos sociales de una nueva memoria espacial deslocalizada y abstracta de tipo "verde". Entra en disputa con la población local, que requiere de recursos como la madera para construir, la leña para combustible, y el agua, las praderas y el bosque para sus animales. También con algunos políticos, y la reticencia de las FFAA por los volúmenes de las mismas, dada la cercanía a la frontera y la fragmentación del territorio nacional. Asimismo, se confronta con grupos empresariales que creen que puede haber una perfecta compatibilidad de cuidado y protección ambiental con actividades comerciales ${ }^{8}$.

Aprovechando esta herencia filosófica territorial, y todo el simbolismo que le irradia, se desarrollan instrumentos económicos paralelos como los mecanismos de pago por servicios ambientales o "nueva economía de la naturaleza" (Fatheur, 2014), que se han venido promoviendo de forma reiterativa en proyectos enfocados a la gestión de

\footnotetext{
${ }^{7}$ En la edición del periódico El Mercurio, del día 14 de agosto de 2018, se indica que en Nueva Zelandia ha habido una importante compra de "remotas haciendas montañosas", consideradas refugios de propietarios ausentes, lo que ha impedido el paso a excursionistas y cazadores locales a desplazarse, restringiendo la posibilidad del disfrute escénico. Esto, ha obligado a agendar en el parlamento una discusión importante sobre este proceso de adquisición de tierras (ver cuerpo A 6).

${ }^{8}$ Los empresarios Ariztía, Matte, Guzmán, Cariola, Cosmelli y Yuraszeck, entre otros, propusieron la compra de la Estancia Chacabuco a puertas cerradas, con 25 mil cabezas de ganado con que contaba en el año 2004. La Estancia estaba valorizada en cerca de 10.000 .000 USD (Diario el Mercurio de Santiago, 25 de julio). Ela cuerdo empresarial habla de un "todos para uno", lo que debe leerse como "todos contra Tompkins".
} 
la biodiversidad. También, se desarrolla un turismo premium o de clase mundial, para visitantes verdes y exclusivos (políticos y actores de fama mundial), que desean salvaguardar su anonimato, y que andan tras las tierras ignotas del fin del mundo, vírgenes y únicas.
La resimbolización corresponde a la forma específica de cómo el capital y sus objetivos se concretan en el espacio (Haesbaert, 2010). Y, más allá de sus propósitos particulares utópicos y eutópicos, se territorializa la adjetivación de territorio "único y exclusivo", dentro del proceso de producción a

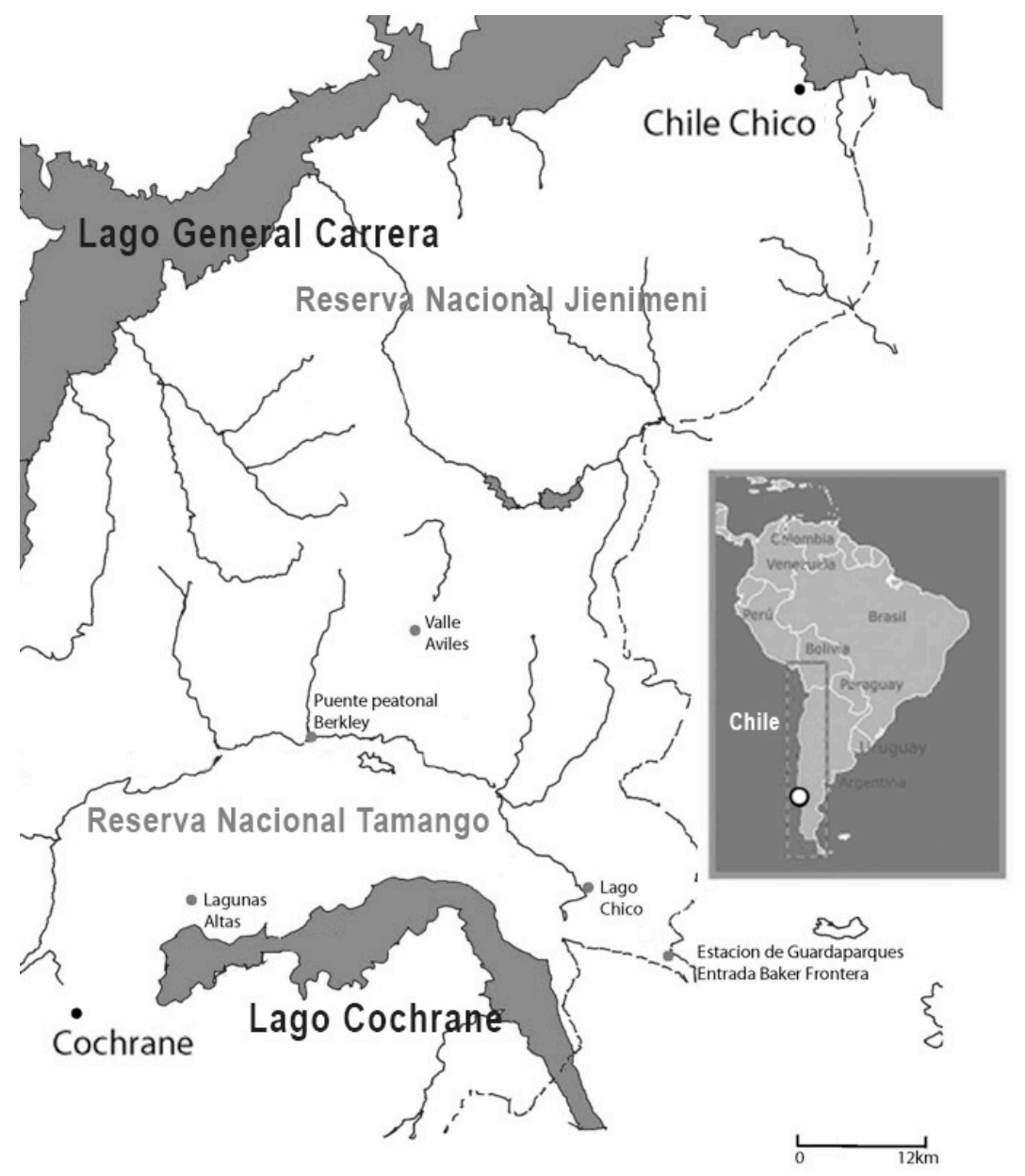

FIGURA 2 - Proyecto de D. Tompkins de creación del gran Parque de la Patagonia, que contempla como conceptos ejes el cambio de producción a conservación y de ganado a vida silvestre, lo que implica la remoción de miles de metros cercos para el libre desplazamiento de todos los animales. FUENTE: elaboración de los autores. 
través de inversiones (i.e. infraestructura, especulación), generando correlaciones de fuerza y de poder con los diferentes actores sociales a través de procesos de control, subordinación e influencia (Bendini \& Steimbreger, 2010). En cualquiera de estos esquemas, la participación local es marginal y subordinada al conservacionismo, ya que no alcanza a beneficiarse dentro de un esquema privatizador de la naturaleza.

Con los predios construye una frontera privada, que altera la línea de reproducción socioespacial de la Agricultura Familiar Campesina (AFC). Fricciona los dispositivos adaptativos del modo de vida, debilitando con el propio control de los recursos de la población. Es decir, el modo tradicional de agricultura descrita por Fabrón \& Castro (2019), que en términos generales define a un conjunto de productores que presentan ciertos rasgos comunes, como el uso de la fuerza de trabajo familiar, la administración y la organización de las actividades agrícolas, un tipo de actividad de carácter principalmente rural, la posibilidad de ser minifundista, una pequeña agricultura comercial o en transición, la máxima utilización de ventajas geo-ambientales en las cuales se ubica y se observa en ella la persistencia de formas tradicionales de producción.

Con evidencia empírica y argumentos ético filosóficos, a través de sus fundaciones (Foundation for Deep Ecology (FDE), Conservation Land Trust (CLT) y Conservation Land Trust-Argentina), y de diversas publicaciones, la compra de tierras que se van adicionando a las de parques nacionales, modifica el espacio fundacional concretizado por el trabajo ganadero, resignificando su valor utilitario y su papel como base material de la reproducción social. Los extensos dominios se convierten en estructuradores de un nuevo orden territorial, que permite la "configuración y producción" de una frontera (Grimson, 2011) que indica límites de posibilidades y usos normados para cualquier vecino y visitante (no pescar, no talar, no introducir animales o fauna y flora exógena). Instalando en sus predios una operación espacial, política-simbólica, que permite la proyección de su propia concepción del mundo, que da nueva forma y valor a la vida silvestre en la Patagonia, irradiándose en cada componente y conjunto del espacio geográfico.

Para el fortalecimiento de biocorredores binacionales de fauna endémica, se imponen restricciones de uso frente a las mercedes de paso a fuentes hídricas, pastizales y bosques para resguardo, lo que viene a confirmar un nuevo elemento de decaimiento a la ganadería ovina y vacuna tradicional. Quedando al margen toda posibilidad de una gobernanza co-participativa y co-gestionada del territorio, es decir, lo relativo a bienes que deben ser administrados para generar prácticas reguladoras entre actores, y que influyen en el curso de las acciones y los resultados en torno a cuestiones territoriales y ambientales (Vallejo-Román \& Rodríguez Torrent, 2020, p. 116). Marginando a las comunidades en la elaboración de un plan para los territorios tradicionales (De Castro et al., 2015; Rodríguez, 2019); y favoreciendo la expansión de otras funciones para los espacios de tipo contemplativo y restaurativo, de viviendas de descanso, de venta de bonos de carbono y turismo exclusivo. Esto significa un incremento en el número de actores externos que se valen de tecnologías y recursos para tener la vivencia y control de lugares de alta biodiversidad y valor ecosistémico, y que sustentados en intencionalidades verdes, en algún punto entrecruzan rentabilidad-contemplación (Vázquez, 2015). 


\subsection{Territorio, crianceros, conservación ambiental y conflictos}

Raffestin (1993) define el territorio como la manifestación espacial de los poderes, como forma de construir relaciones sociales entre actores y ocupaciones, integrando a los que cuentan con tenencia y sin tenencia, los legales e ilegales, los establecidos y los errantes. De modo que, la territorialización, como capacidad semántica y de dominio como el de la ecología profunda, se vuelve una estrategia fundante al limitar el uso del espacio por un sujeto (Barañano, 2005), lo que crea un "nuevo lugar" (Vallejo-Román et al., 2020, p. 117), ya que "la acción social es política cuando pretende controlar o influir las decisiones relativas a los asuntos públicos" (Balandier, 1969, p. 36).

La directriz política, normativa y administrativa, afecta el núcleo de la cultura rural, ya que la ecología profunda es un programa explícito de autoridad, poder y modelo de organización para su implementación. Lo que produce una subordinación de las formas locales de conocimiento ante las fundaciones que operan con participación de científicos europeos, chilenos y argentinos, que sostienen la idea de una geomarca Patagonia, como lugar único en términos ecosistémicos.

El territorio se vuelve esencialmente híbrido. Resultado de la apropiación y valorización del espacio mediante la representación y el trabajo [y que] no se reduce a un mero contenedor de los modos de producción y de la organización del flujo de mercancías, capitales y personas, sino también es un significante denso de significados y un tupido entramado de relaciones simbólicas (Giménez, 1999, p. 27). El nuevo significante se convierte en fundamento de un reclamo de identidad local ("siempre hemos vivido aquí y trabajado aquí"), al verse restringidas las lógicas productivas tradicionales por la modificación de límites. Ya que, si bien éstos pueden ser alterados por el ser humano o por eventos ajenos a éste (en el caso de las fronteras naturales), el territorio permanece y prevalece como durabilidad generadora de identidad socio-espacial (Martínez et al., 2015, p. 32).

\subsection{Identidad, ritualidad de las $A F C y$ vocación territorial}

Si afirmamos que el territorio de la AFC de la Patagonia está conformado por las diversas infraestructuras y las acciones humanas que han sido puestas sobre el espacio habitado, vivido y construido históricamente, constituye su marco de posibilidad para la realización de la vida y su modo de ser. Expresa una comprensión material y simbólica del mundo que se manifiesta como modo de vivir la vida (ritualidad anual y estacional), traducida en la constitución de la existencia física, social y simbólica. "Constitutiva y construida" - dirá Sosa (2012) -, ya que marca las decisiones y relaciones de poder puestas sobre él, permiten su escritura, formas de representación (etic) y construcciones locales (emic).

Las espacialidades concebidas, contradictorias y en disputa, entre una productiva y no productiva (en el uso ordinario del término), revela una forma de producción del territorio, de control y soberanía, compuesta por distintos elementos que permiten hablar de los mundos que contiene y los movimientos de la vida. En este caso expresan una articulación geo-eco-antrópica, que da paso a dimensiones 
políticas, económicas, culturales y ambientales (Sosa, 2012).

Etnográficamente, entre los crianceros una oveja es considerada productiva entre 4 a 7 años, y su vida como productora depende de su dentadura para poder alimentarse autónomamente. El ciclo anual comienza con la parición de las ovejas en primavera, cuya carne puede ser vendida en el mismo año. Las hembras no sacrificadas (su mayoría), son destinadas a la reproducción, mientras los machos son castrados. Los llamados "capones" son importantes como productores de lana, pero solo cuando hay poder comprador. Luego, a los dos meses, los animales son marcados (señalada) como signo de propiedad en el mismo momento que se capa a los machos. Cuando se trata de importantes rebaños, es el primer momento de la cuenta, lo que permite hacer la raya para la suma de una buena o mala temporada, ya que la ecuación favorable es entre el número de ovejas que son separadas para los carneros reproductores. A este proceso, le sigue la esquila; $y$, enseguida, se realiza el baño para liberar a la majada de los ectoparásitos (sarna ovina). Ahí, los animales vuelven al campo, los nuevos ejemplares son destetados y separados por su condición de fértil, borrego/a, carneros, corderos y capones. A estas faenas le acompañan la restauración de cercos, los cuidados de la majada, la atención permanente a las condiciones climáticas para protección frente a las inclemencias del clima (mover a resguardo) y frente a los depredadores, así como la cadena de producción, requiere de alternancia en el uso de los pastizales. Al final, la venta será otro proceso?.

El territorio criancero como espacio cultural, revela todas las referencias de una actividad ininterrumpida, propias de las acciones individuales y colectivas reiteradas por generaciones. Es un contenedor de la experiencia colectiva; un horizonte simbolizado y apropiado en el uso, expresado en una temporalidad que desde el presente va hacia el pasado y hacia el futuro; $y$, es un recurso de poder que puede activarse como memoria, ya que es un bien legítimo que se disputa en pequeñas fracciones o grandes extensiones (Toboso \& Valencia, 2008). Corresponde al marco social de la geografía de la vida cotidiana, donde se insertan las unidades de producción, los procesos de trabajo que dan forma a una red, ordenada y simbólica de la vida que excede el mundo laboral.

El conflicto se vuelve evidente: es sobre la definición de la vocación territorial. Se crea una nueva territorialidad tensionada, que excede lo local. Se multiplica una sensibilidad que genera adhesión en la opinión pública nacional e internacional (i.e. "Patagonia sin Represas"; "Reserva de vida"), generando una conciencia sobre la idea de "conservación de la vida", que también presiona sobre las autoridades políticas nacionales y regionales. Sin embargo, las posiciones defendidas por Tompkins (reserva de vida bajo postulados de la ecología profunda) y los crianceros (uso tradicional), configuran de manera contradictoria la síntesis de las experiencia comprehensiva en los

\footnotetext{
${ }^{9}$ La que estimaba -durante la primera mitad del siglo XX, y por las características de los caminos-, que una distancia límite de rentabilidad por volumen, no podía exceder de unos 100 kilómetros desde el lugar de producción hasta un puerto de embarque (Rodríguez et al., 2019). También existen una serie de factores reproductivos que se ubican fuera de la lógica de la manipulación, y que hablan de un equilibrio precario, como el frío, los vientos, la nieve y la falta de alimentos.
} 
distintos planos, ya que ambas se hacen cargo de la biodiversidad existente, construyendo relaciones físicas, genéticas y ecosistémicas, que dan lugar al medioambiente: "el ámbito biofísico natural y sus sucesivas transformaciones artificiales así como su despliegue espacial" (Sunkel, 1980, p. 13).

Lo más crítico y conflictivo territorialmente es la expulsión de la fauna exógena (ovejas y vacunos), ya que es la base de la economía rural patagónica que sostiene el poblamiento interior del sur de la región de Los Lagos y de Aysén. Tompkins, creía que la población activa "se podía reconvertir" y "asalariar" dentro de la figura de los parques, porque podían generar "muchos empleos estables y con ingresos mejores" que los que proporciona su actividad criancera (entrevista a un administrador de un predio de D. Tompkins, 2016). El impacto es mucho más significativo; lo que se altera son las lógicas espaciales extensivas de la especialización, que concilian la autonomía, la ritualidad del trabajo, las necesidades de demanda de bienes y servicios, las actividades para la generación de la renta y la construcción de las redes y relaciones sociales con sus referentes identitarios (Sili, 2007, p. 11). Al respecto, señala un criancero en Puerto Ibáñez, región de Aysén:

"Yo estaba aserrando madera aquí [en mi casa], y llegó
Tompkins. Él venía a comprar mi tierra. Conversa-
mos y conversamos mucho. A él le interesaba lo que
yo tenía, que no es tanto la tierra. Porque cuando se
acaba el agua aquí abajo, yo subo varias horas con los
animales, y siempre tengo agua en un lugar específico.
En los años más secos siempre hay agua ahí. Eso es
puro conocimiento, si las tierras no es tanto lo que
valen. Entonces, si uno vende, se tiene que ir. Pero,
¿a dónde? Si todo lo que uno tiene y es, está aquí...
Uno es de aquí, no es de otro lado. Y no puede aceptar
que le vengan a decir cómo tiene que hacer las cosas.
Esto es puro trabajo, todos los días. Entonces, como uno en esto [del trabajo] se la arregla solo [sin ayuda del Estado], muchos se tientan y venden. Yo le dije que no, pero él sabía que yo tenía agua, y eso era lo que quería".

\section{Análisis: Repensando la sustentabilidad en la Patagonia chilena del S. XXI. Lenguajes de valoración y territorio en disputa}

Se presentó una mirada para entender los territorios y sus configuraciones, y la posibilidad de observación de las distintas lógicas espaciales y de los esfuerzos localizados que las comunidades realizan para alcanzar su reproducción en el tiempo. Asimismo, el peso de las hegemonías semánticas o lenguajes de valoración que se ponen sobre él.

Etnográficamente, lo importante es el holismo subyacente. La AFC de la Patagaonia-Aysén se establece conforme a ciertas capacidades de los productores de apropiarse de un espacio, a través de la movilidad predial entre el espacio doméstico al peri-doméstico, así como con los espacios extra-prediales en una ruralidad dispersa o hacia los agrupamientos (Comerci, 2010, p. 86). Fórmula cualitativa, material y simbólica, anclada en determinados procesos cíclicos como el ganadero, la producción de leña con fines de uso doméstico o comercial, o madera aserrada para fines de construcción, y que proporcionan identidad y coherencia.

La operación material, simbólica y política del control de grandes extensiones de ecosistemas, para la creación de grandes "biocorredores" que dan forma a un parque privado de conservación, produce una reconfiguración al introducir un nuevo 
"lenguaje de valoración" (Martínez Alier, 2014). La lógica de, "a espacios privados, reglas privadas" (diferenciando tierras en descanso y tierras sujetas a acción productiva), supera los marcos de acción del propio mundo del trabajo especializado, al irradiarse y desbordarse hacia otras dimensiones de la vida social, redefiniendo la propia capacidad y poder para frenar el cambio o ejercer derechos sobre usos. Con menos tierras y bosques por donde errar con el ganado y protegerlo de las inclemencias climáticas, y sin madera para talar y aserrar, lo que se pone en juego entre los lenguajes de valoración, es el universo cognitivo que permite hablar del "nosotros pionero o colono", que construyó la espacialidad patagónica. Y, la conciencia de la construcción del hábitat, abre la posibilidad de activar la memoria colectiva para reapropiarse y revalorizar los recursos locales, y, ponerlos en el centro de la disputa política como cuestión de derecho, adhesión a una historia común y como contra-narrativa de los bienes comunes.

A partir de su savoir faire, se produce una "politización del sentido" del territorio (Porto-Gonçalves, 2009), al incorporarse un conjunto de componentes que permiten construir desde el lugar ocupado en la estructura social y territorial, discursos de identificación local, densificando el territorio con elementos particulares para dar sentido comunitario y para construir un territorio cultural distinto al de la ecología profunda. Se deconstruye así, la idea del hombre solitario con las ovejas, y la figura de lo legal y lo ilegal, para transformar las restricciones impuestas en lo legítimo y justo, revindicando como derecho fundamental no sólo los recursos, sino el derecho a permanecer bajo condiciones preexistentes que otorga el haber llegado primero.

La inscripción territorial de los reclamos contra las áreas protegidas incorpora el problema del acceso a los recursos para la sostenibilidad de la propia vida (agua, tierra, recursos forestales, acceso a la infraestructura básica), en un doble sentido: como disputa económica y como conjunto de valores culturales e identitarios. Si aceptamos este reclamo, frente a un interlocutor privado que limita las condiciones de acceso, se entenderá que la consigna "fuera Tompkins", es la denuncia que habla de un teatro-territorio producto de un conflicto socioambiental o topografía conflictiva ${ }^{10}$, ya que ontológicamente la definición de lo verde -que está definiendo un parque privado- desencadena la incertidumbre misma de su sobrevivencia.

Una unidad familiar campesina necesita de 4, 5 o más hectáreas para tener un vacuno; requiere de un año al menos para vender su ganado; demanda de agua, pasto y cobijo en el bosque; y, disminuir los riesgos de amenazas en la cadena trófica. Mientras, por el otro lado, el parque privado expulsa la masa ganadera, quita los cercos, con el consecuente aumento de los depredadores endémicos como el puma; pero, sobre todo desorganiza la estructura espacial que permite la reproducción de la unidad económica que requiere de grandes extensiones para la movilidad del ganado, para permitir simultáneamente la reproducción biológica y cultural de la unidad doméstica.

Confrontadas las lógicas de la ecología profunda y el derecho privado de propiedad frente a geografía de la vida cotidiana de la AFC, nos parece

\footnotetext{
${ }^{10}$ Entendemos la noción de "topografía conflictiva" como aquello que está fuera del espacio "en sí", y que más bien se ubica en el espacio de la representación. Lo conflictivo es el efecto de una relación dispar de lógicas y de poder.
} 
apropiada la idea de "configuración cultural" propuesta por Gundermann et al. (2018), para definir a la población especializada. No es posible postular a priori una unidad o colectivo, a pesar que no hay diversificación productiva entre las unidades familiares, sino que dentro de la heterogeneidad de los productores de ganado y leñadores (i.e. número de animales, cantidad de tierra, cercanía de caminos principales), es la amenaza "al pionero" y "al colono" la que genera elementos simbólicos de consenso, lo que favorece una representación de identidad que aglutina dentro de la escala local. Es decir, por sobre todo, frente a la amenaza a la propia tradición, producto de la creación de mega parques, prevalece un conjunto de elementos unificadores que se manifiestan como:

“construcciones ideáticas que sobre una realidad social concreta elaboran determinados sectores sociales, [los que] apoyándose en hechos reales o mitificados, pretenden velar... [y] ofrecer unos referentes de identificación al conjunto de individuos que ocupan un territorio determinado" (Palenzuela \& Hernández, 1995).

El conflicto territorial entre los parques privados de D. Tompkins (ahora traspasado a través de sus fundaciones al Estado) y los pequeños productores, es sobre los valores y prioridades puestos en el territorio. Como caso de estudio, debe leerse como la posibilidad de una reflexión teórica sobre la reescritura territorial y sus efectos; o, cómo el espacio se vuelve territorio, a partir de los usos y el control en los marcos de una condición polar entre sustentabilidad "débil" (O'Connor \& Martínez Alier, 1998) y una "fuerte" (Daly \& Cobb, 1989; Daly, 1991). Esto es, si el mercado o la mantención de statu quo, son suficientes, sumado a los avances en tecnología, para la sostenibilidad; o, si se requieren intervenciones directas con importantes cambios institucionales. En ambas perspectivas se juegan dos cosas: 1) la capacidad de substitución del capital natural; 2) la condición correctiva y de intervención, que implica la introducción de elementos de mayor justicia y equidad (dimensión fuerte). En este sentido, la propuesta de Tompkins estaría más allá de esta figura, ya que fragmenta el espacio tradicional de la vida cotidiana que permite que pueda darse la reproducción cultural y generacional, tal como se dio en las concepciones clásicas de los parques nacionales en Europa y Estados Unidos de Norteamérica.

Frente a lo señalado, nos parece que tres puntos importantes son los que vuelven a la Patagonia significativa desde el análisis antropológico:

\section{Primero,}

sin alardes nacionalistas de ninguna naturaleza, que un extranjero sea "capaz de fragmentar el territorio nacional" a través de una prenoción sobre la naturaleza, lo que implica una queja de las Fuerzas Armadas sobre las fracturas territoriales por encontrarse en zonas fronterizas; b) que un empresario sea un empresario "verde", en el país de los buenos negocios (o donde todo es lucro); y, c) que con la compra de tierras se restrinjan actividades tradicionales de la población local y el potencial desarrollo de otras ligadas a empresarios nacionales, sin pretensión de defensa de éstos últimos.

\section{Segundo,}

lo que se impone y que genera adhesiones extra locales de tipo proteccionista, es que produce una organización distinta del espacio a través de su re- 
simbolización, por medio del derecho a la existencia de todos los seres y sin jerarquía, lo que pone en evidencia los usos diferenciados que esta zona del país puede tener, siempre conforme a posibilidades de poder y resistencia. Lo que marca el hastío ciudadano al modelo extractivista.

\section{Tercero,}

pese a la extraordinaria belleza de la bioregión, la escasa atención que ha tenido la zona para la generación de un sistema de oportunidades y trabajo de tipo local que favorezca el arraigo, ya que su demografía es equivalente a 0.8 o 0.9 habitantes por kilómetro.

Cualquier orden de conflictos topográficos y de lenguajes de valoración en torno a derechos y legislación, siempre está cruzado por elementos políticos de gobernanza territorial. Ya que se instalan y ponen en práctica de formas de gestión y planeación del territorio, que implican modos de participar, organizarse y gestionar, debiendo distinguirse la autoridad y el poder. En este caso, debemos reconocer que existe una decisiva influencia del dinero para crear nuevas condiciones de reorganización del territorio y crear nuevos mapas de sentido de carácter extra local. La capacidad de movilizar capitales define una conflictividad constitutiva de su reconfiguración, porque las nuevas formas de trabajo y tenencia (i.e. con orientación al turismo, la energía, la especulación, venta de bonos de carbono, condominios y membresías) alteran la vida tradicional, al verse fractalizada en sus ciclos. El concepto y materialización del gran parque privado de conservación bajo claves de ecología profunda, sumado a las áreas protegidas por parte del Estado, se impone por sobre la especificidad de una condición relacional consolidada por los antiguos colonos y que se movía con cierta infinitud de recursos en estepas que parecían infinitas y bosques de protección. Esta forma, planteada como ontología, y que ha sido propuesta por Arturo Escobar (2015, p. 29-33), puede "definirse como aquella en que nada (ni los humanos ni los no humanos) preexiste a las relaciones que nos constituyen. Todos existimos porque existe todo". Por lo que los territorios se convierten simultáneamente en "espacio-tiempos, tanto de la comunidad y de interrelación de lo que circunda la experiencia, estableciendo sinergias positivas para humanos y no humanos".

La propuesta de Tompkins, que constituye una forma de marcar el pasado al identificarlo con degradación por sobrepastoreo, y el presente como reserva ecosistémica, alcanza mayor adhesión y reputación en circuitos globales y en ámbitos extralocales más cercanos a valores posmaterialistas como los propuestos por Inglehart (1997). De modo que, en algunos grupos más bien genera resistencias locales, porque hay una disparidad y asimetría en los beneficios sociales esperados en la región; hay muchos abismos entre las expectativas y dividendos esperados por operadores turísticos que se ven favorecidos por estas nociones proteccionistas que consolidan la idea de una geomarca internacional Patagonia (Rodríguez et al., 2014), y población especializada en la ganadería o el trabajo maderero que tiene sus propios ciclos económicos y que requiere de grandes extensiones para su reproducción como cultura del trabajo. Confrontados los lenguajes de valoración, por una parte, lo verde propuesto por Tompkins, se expande; por otra, desde la población económicamente especializada, se lee como una contracción, como un flujo de violencia que pone en riesgo la propia existencia. 


\section{Conclusiones}

Lo que hemos desarrollado en este análisis territorial dentro de los lenguajes de valoración, es una discusión que podría ayudar a los países y a las comunidades a definir su matriz agraria en relación a qué producir y cómo conseguirlo. Por una parte, se trata de cómo abordar la condición local como parte del aparato productivo regional y nacional. Por otra, corregir lo que pueda considerarse ambientalmente nocivo, porque desde la posición de la AFC se puede impulsar un modelo de corte agroecológico, coherente con la crisis ambiental que sacude al planeta, ayudando también a la sostenibilidad de las culturas del trabajo locales.

Esta segunda perspectiva introduce dos elementos centrales para los procesos territoriales: 1) la idea de justicia; y, 2) la consideración de los derechos humanos. La primera, porque es una forma de oponerse a la privatización de los bienes colectivos como las semillas, la tierra, el bosque y el agua. Y, la segunda, porque asegura dos derechos: a la alimentación y a permanecer en el territorio. Como bien sostiene Arturo Escobar: "las luchas por los territorios se convierten en lucha por la defensa de los muchos mundos que habitan el planeta" (2015, p. 29), y ello entraña varios derechos: a ser, o el de la identidad; el de vivirla en un territorio; a ser y expresar de una manera particular; $y$, a sostener una idea del futuro (Escobar, 2015, p. 32). Ello, también ayuda a explicar cómo se clasifican frente a otros (De La Cadena, 2015), y es lo que garantiza la posibilidad de supervivencia, de la población local y del pluriverso.

Es de destacar en la argumentación desarrollada que se enfrenta la disputa territorial como problema de ecología política. Pero, finalmente debe ser abordada como un campo relacional en el que confluyen la gobernanza, las subjetividades y los conocimientos, lo que ha estado tanto del lado de las construcciones filosóficas como la ecología profunda, del pragmatismo del Estado y de la agricultura familiar campesina. En todas estas vertientes de pensamiento están presentes formas de poder, derecho e imaginación para estructurar el campo territorio, como espacio de la diferencia en el que es imposible pensar el uno sin el otro o los otros (Escobar, 2015; de la Cadena \& Starn, 2009). En esta inflexión y disputa disonante entre valores y pragmatismo, la naturaleza adquirirá su dimensión política, ficcional y performativa, constituyendo un recurso retórico, mitológico y epistémico desde dónde es posible establecer una negociación con el mundo. Lo político, fluye como ejecución de decisiones; desplegándose comunicacionalmente más allá del territorio autocontenido, convirtiéndose este territorio en una comunidad política como nunca en su historia.

Quizá, por primera vez, hay consciencia entre los pequeños productores del valor alcanzado por estas "tierras olvidadas" y de la propia existencia en cuanto sujetos. Pero, ahora la memoria como experiencia multigeneracional lo es dentro de un modo de producción distinto y con una apertura al mundo también diferente. Estos nuevos trabajadores son un producto del presente: de las luchas planetarias que se dan dentro de la deep ecology, shallow ecology, los movimientos sociales ambientalistas y la consciencia de los límites del planeta, que son también parte de la Patagonia del siglo XXI. 


\section{Referencias}

Agrawal, A.; Redford, K. Conservation and Displacement: An Overview. Conservation and Society, 7, 1-10, 2009. doi: 10.4103/0972-4923.54790

Balandier, G. Antropología Política. Barcelona: Ediciones Península, 1969.

Barañano, A. Diccionario de relaciones interculturales, diversidad y globalización. Madrid: Editorial Complutense, 2005.

Bendini, M.; Steimbreger, N. Dinámicas territoriales y persistencia campesina: redefinición de unidades y espacios de trabajo de los crianceros en el norte de la Patagonia. Revista Transporte y Territorio, 3, 59-76, 2010.

Braidotti, R. Lo posthumano. Barcelona: Gedisa, 2015.

Brockington, D.; Igoe, J. Eviction for Conservation: A Global Overview. Conservation and Society, 4(3), 424470, 2006.

Comerci, M. E. Tenemos que ir allá y pegar la vuelta. Continuidades y cambios en las prácticas de movilidad campesinas en contextos de conflictividad emergente. Revista Transporte y Territorio, 3, 77-102, 2010.

Coronato, F. Le rôle de l'élevageovindans la construction du territoire de la Patagonie. Autre [q-bio.OT]. Français: AgroParisTech, 2010.

Daly, H. E.; Cobb, J. B. For the Common Good: Redirecting the Economy Towards Community, The Environment, and a Sustainable Future. Boston: Beacon Press, 1989.

Daly, H. Commentary: Toward some operational principles of sustainable development. Ecological Economics, 2, 1-6, 1991.

De Castro, F.; Hogenboom, B.; Baud, M. Introducción. Gobernanza ambiental en América Latina en la encrucijada, moviéndose entre múltiples imágenes, interacciones e instituciones. En: de Castro, F.; Hogenboom, B.; Baud, M. (Coords.). Gobernanza ambiental en América Latina. Buenos Aires: CLACSO, p. 13-38, 2015.

De La Cadena, M.; Starn, O. Indigeneidad: problemáticas, experiencias y agendas en el nuevo milenio. Tabula Rasa, 10, 191-223, 2009.

De La Cadena, M. Earth Beings. Ecologies of Practice Across Andean Worlds. Durham: Duke University Press, 2015.

Del Risco, E. La conservación y los jardines botánicos. Revista del Jardín Botánico Nacional, 3, 167-195, 1982.

Demerit, D. What is the social construction of nature? A typology and sympathetic critique. Progress in Human Geography, 26(6), 767790, 2002. doi: 10.1191/0309132502ph402oa

Descola, P.; Gisli, P. Introducción. En: Descola, P.; Gísli, P. (Eds.). Naturaleza y sociedad. Perspectivas antropológicas. México: Siglo Veintiuno, 1133, 2001.

Durand, L. Naturalezas desiguales. Discursos sobre la conservación de la biodiversidad en México. México: UNAM, 2017.

El Mercurio. Hoy se decide futuro de codiciada estancia. Viernes 2 de julio. Santiago, 2004.

Escobar, A. Territorios de diferencia: la ontología política de los discursos del territorio. Cuadernos de Antropología Social, 41, 25-38, 2015.

Fatheur, T. Nueva economía de la naturaleza. Santiago: Ediciones Böll, 2014.

Ferrero, B. Tras una definición de área protegida. Apuntes sobre la conservación de la naturaleza en Argentina. Revista Universitaria de Geografia, 27, 99-117, 2018.

Ferrero, B. et al. Definiendo la Conservación: el caso del Parque Nacional Iguazú, Argentina. Ecología Política, 46, 85-89, 2013.

Giménez, G. Territorio, cultura e identidades. La región socio-cultural. Estudios sobre las Culturas Contemporáneas, 9, 25-57,1999.

Grimson, A. Los límites de la cultura. Crítica de las teorías de la identidad. Buenos Aires: Siglo XXI Editores, 2011.

Grimson, A.; Merenson, S.; Noel, G. Antropología ahora. Debates sobre la alteridad. Buenos Aires: Siglo XXI Editores, 2011. 
Guha, R.; Gadgil, M. Los hábitats en la historia de la humanidad. Ayer, 11, 49-110, 1993.

Gundermann, H.; González, H.; Durston, J. Interetnicidad y relaciones sociales en el espacio atacameño. Estudios Atacameños, 57, 161-179, 2018. doi: 10.4067/S071810432018005000501

Haesbaert, R. O mito da Desterritorialiçao. Do "Fim dos Territórios" à Multiterritialidade. Río de Janeiro: Bertrand Brasil, 2010.

Hardin, G. The Tragedy of the Commons. Science, 162(3859), 1243-1248, 1968.

Hayles, N. K. How We Became Posthuman: Virtual Bodies in Cybernetics, Literature, and Informatics. Chicago: The University of Chicago Press, 1999. http://aleph.unisg.ch/ hsgscan/hm00163662.pdf.

Inglehart, R. Modernization and Postmodernization: Cultural, Economic and Political Change in 43 Societies. Princeton, N. J.: Princeton University Press, 1997.

Kuppe, R. Indígenas y medio ambiente: conservacionismo a espaldas de los guardianes de la tierra. Boletín de Antropología Americana, 35, 95-104, 1999.

Martínez Alier, J. El ecologismo de los pobres. Conflictos ambientales y lenguajes de valoración. Santiago: Quimantú, 2014.

Martínez, E.; Matthew L.; Salas, A. Reorganización del territorio y transformación socioespacial rural-urbana. México: ISS-UNAM/Bonilla Artigas, 2015.

Naess, A. The Third World, Wilderness, and Deep Ecology. En: Sessions, G. Deep Ecology for the twenty-first Century. Boston: Shambala, p. 397-407, 1995.

Núñez, A.; Miranda, F.; Aliste, E.; Urrutia, S. Conservacionismo y desarrollo sustentable en la geografía del capitalismo: negocio ambiental y nuevas formas de colonialidad en la Patagonia-Aysén. En: Núñez, A.; Aliste, E.; Molina, R. (Comp.). (Las) Otras Geografias en Chile. Perspectivas sociales y enfoques críticos. Santiago: LOM, p. 23-46, 2019.

O’Connor, M.; Martínez Alier, J. Ecological Distribution and Distributed Sustainability, En: Faucheux, S.; O'Connor, M.; Van der Straaten, J. (Ed.). Sustainable Development:
Concepts, Rationalities and Strategies. London: Kluwer Academic Publishers, p. 33-53,1998.

Ostrom, E. Comprender la diversidad institucional. Oviedo: krk, 2013.

Ostrom, E.; Walker, J. Trust and reciprocity. Interdisciplinary lessons from experimental research. New York: Russell Sage, 2003.

Palenzuela, P.; Hernández, J. Poner Monachil en el mapa. Estudio antropológico de un proceso de transformación cultural, Granada. Granada: Universidad de Granada-Diputación Provincial de Granada, 1995.

Porto-Gonçalves, C. W. De saberes y de territorios: diversidad y emancipación a partir de la experiencia latino-americana. Revista de la Universidad Bolivariana, 8(22), 121-136, 2009.

Raffestin, C. Por uma geografia do poder. San Pablo: Atica, 1993.

Rivera, C.; Vallejos-Romero, A. La privatización de la conservación en Chile: repensando la gobernanza ambiental. Revista Bosque, 36(1), 15-25, 2015.

Rodríguez, J. C.; Sáenz, J.; Gissi N.; Mandujano, F. Fronteras internas y hegemonías predicativas en Chile: el caso de la Patagonia. Chungara. Revista de Antropología Chilena, 50(4), 633-650, 2018.

Rodríguez, J. C.; Sáenz, J.; Gissi N.; Mandujano, F. Ruralidad y 'frontera interior' en la Patagonia chilena: unidades domésticas, vida cotidiana y nuevos ejes para su comprensión. Revista Magallania, 47(1), 41-63, 2019.

Rodríguez, J.; Reyes, S.; Medina, P. Territorio, paisaje y marketing global. Imaginarios en la construcción de la Patagonia como marca. Revista Magallania, 42(2),109-123, 2014. doi: 10.4067/S0718-22442014000200006

Rodríguez, T. Gobernanza ambiental en cuencas transfronterizas: la cuenca del río Sixaola (Costa Rica - Panamá). Iztapalapa Revista de Ciencias Sociales y Humanidades, 87(40), 71-99, 2019.

Sánchez, G. La Patagonia Vendida. Los nuevos dueños de la tierra. Buenos Aires: Marea Editorial, 2006.

Santamarina, C. B. De parques y naturalezas. Enunciados, 
cimientos y dispositivos. Revista de Dialectología y Tradiciones Populares. LXVI(1), 297-324, 2009. doi: 10.3989/ rdtp.2009.019

Sessions, G. The Deep Ecology Movement: A Review. Environmental Review, 11(2), 105-125, 1987.

Sili, M. Fragmentación territorial y desarrollo rural. Nuevas interpretaciones y propuestas para el desarrollo de los territorios rurales en un contexto de globalización. Serie Desarrollo Sustentable, Documento de Trabajo 1, 2007.

Sosa, M. ¿Cómo entender el Territorio? Guatemala: Editorial Cara Parens, 2012.

Sunkel, O. Introducción. En: Sunkel, O.; Gligo, N. (Selecc.). Estilos de desarrollo y medio ambiente en la América Latina, 9-64. México: F.C.E., 1980.

Toboso, M.; Valencia, G. Una representación discursiva del espacio tiempo social. Estudios Sociológicos, 1, 119137, 2008.

Vallejo Román, J.; Rodríguez Torrent, J. C. Bienes comunes, conservación ambiental y economía campesina. Un estudio de caso en el Parque Nacional Cofre de Perote, Veracruz, México. Polis. Revista Latinoamericana, 57, 115-137, 2020. doi: $10.32735 /$ S0718- 6568/2020-N57-1567
Valles, M. Técnicas cualitativas de investigación social. Reflexión metodológica y práctica profesional. Madrid: Editorial Síntesis, 1999.

Vázquez, A. D. Lógicas espaciales campesinas en la estepa patagónica. Magallania, 45(2), 273-297, 2017.

Vázquez, A. D. Lógicas territoriales y discursos inmobiliarios en ámbitos rurales patagónicos semiáridos. Confusión social e incertidumbres geográficas. Revista de Informes Científicos Técnicos ICT-UNPA, 7(1), 244-260, 2015.

Link

https://www.youtube.com/watch?v=In2bdaN3QdI Entrevista a Douglas Tompkins www.elpost.cl parte 1, 2, 3, 4.

https://www.youtube.com/watch?v=bQc_DrC4rJk Creando el Futuro Parque Nacional Patagonia (HD)

Videos

Mountain of storns: https://www.youtube.com/watch?$\mathrm{v}=$ Rhtq8rYeLz4

$180^{\circ}$ South: https://www.youtube.com/watch?v=mqhh7c1SUHY 